\title{
Identification of interaction partners of the adapter protein Nck in T cells
}

\author{
J Pieper*1, B Lengl-Janßen ${ }^{1}$, M Voss ${ }^{1}$, C Gelhaus ${ }^{2}$, M Leippe ${ }^{2}$, O Janssen ${ }^{1}$ and \\ M Lettau ${ }^{1}$
}

\author{
Address: ${ }^{1}$ University Hospital Schleswig-Holstein, Campus Kiel, Institute of Immunology, Kiel, Germany and ${ }^{2}$ Department of Zoophysiology, \\ Zoological Institute, Christian-Albrechts University, Kiel, Germany \\ * Corresponding author
}

\author{
from 12th Joint Meeting of the Signal Transduction Society (STS). Signal Transduction: Receptors, Mediators and Genes \\ Weimar, Germany. 29-3I October 2008 \\ Published: 26 February 2009 \\ Cell Communication and Signaling 2009, 7(SuppI I):A84 doi:I0.II86/I478-8IIX-7-SI-A84
}

This abstract is available from: http://www.biosignaling.com/content/7/SI/A84

(c) 2009 Pieper et al; licensee BioMed Central Ltd.

The adaptor protein Nck almost exclusively consists of one SH2 domain and three SH3 domains and plays an important role in linking receptor tyrosine kinases (and associated proteins) to modulators of the actin cytoskeleton. In T cells, Nck thereby connects T cell receptor signaling to the machinery of actin reorganization thus initiating changes in cell polarity required for T cell effector function. We previously showed that Nck interacts with the death factor FasL and is required for the recruitment of FasL to the cytotoxic immunological synapse upon recognition of a target cell (Lettau et al., PNAS 2006). Given the importance of Nck in T cell effector function, we now performed a systematic screening for interaction partners of the four individual interaction modules of Nck in primary and leukemic T cells. To this end, we expressed full length Nck, the three SH3 domains and the individual SH3 and $\mathrm{SH} 2$ domains of Nck as GST fusion proteins to precipitate binding partners from untreated or pervanadate-treated PHA blasts, Jurkat and HUT78 cells. Candidate binding proteins were cut from gels after Coomassie, Silver, Sypro Ruby or Flamingo Pink staining and processed by tryptic in gel digestion for mass spectrometrical analysis. Of several candidate-binding partners identified by the pulldown assays, we confirmed direct interaction by Far Western Blotting. As might be expected, we observe major differences in Nck binding proteins from resting versus activated $\mathrm{T}$ cells. Interestingly, the list of interactors not only points to known functions including recruitment of the WASP/Arp $2 / 3$ complex and the actin cytoskeleton but also to the recently described nuclear translocation and function of Nck, e.g. in damaged cells. Therefore, we are currently focusing on the verification of the latter Nck interactions in nuclear extracts to unravel this new aspect of Nck biology. 